\title{
Researching the researchers: psychological distress and psychosocial stressors according to career stage in mental health researchers
}

\author{
Nicole T. M. Hill ${ }^{1,2,3^{*}}$, Eleanor Bailey ${ }^{4,5}$, Ruth Benson ${ }^{6,7}$, Grace Cully $^{6,7}$, Olivia J. Kirtley ${ }^{8}$, Rosemary Purcell ${ }^{4,5}$, \\ Simon Rice ${ }^{4,5}$, Jo Robinson ${ }^{4,5}$ and Courtney C. Walton ${ }^{4,5}$
}

\begin{abstract}
Background: Although there are many benefits associated with working in academia, this career path often involves structural and organisational stressors that can be detrimental to wellbeing and increase susceptibility to psychological distress and mental ill health. This exploratory study examines experiences of work-related psychosocial stressors, psychological distress, and mental health diagnoses among mental health researchers.
\end{abstract}

Methods: This international cross-sectional study involved 207 mental health researchers who were post-graduate students or employed in research institutes or university settings. Work-related psychosocial stressors were measured by the Copenhagen Psychosocial Questionnaire III (COPSOQ III). Psychological distress was assessed using the Depression-Anxiety-Stress Scale-21 (DASS-21). Thoughts of suicide was assessed using an adaptation of the Patient Health Questionnaire-9 (PHQ-9). History of mental health diagnoses was assessed through a custom questionnaire. Pearson's chi-square test of independence was used to compare mental health diagnoses and suicidal ideation across career stages. The association between work-related psychosocial stressors and psychological distress was conducted using multivariate linear regression controlling for key demographic, employment-related and mental health factors.

Results: Differences in 'demands at work' and the 'work-life balance' domain were lowest among support staff $(p=0.01)$. Overall, $13.4 \%$ of respondents met the threshold for severe psychological distress, which was significantly higher in students compared to participants from other career stages $(p=0.01)$. Among the subgroup of participants who responded to the question on mental health diagnoses and suicidal ideation $(n=152), 54 \%$ reported a life-time mental health diagnosis and $23.7 \%$ reported suicidal ideation since their academic career commencement. After controlling for key covariates, the association between the 'interpersonal relations and leadership' domain and psychological distress was attenuated by the mental health covariates included in model $3(\beta=-0.23, p=0.07)$. The association between the remaining work-related psychosocial stressors and psychological distress remained significant.

Conclusions: Despite working in the same environment, research support staff report experiencing significantly less psychosocial stressors compared to postgraduate students, early-middle career researchers and senior researchers. Future research that targets key modifiable stressors associated with psychological distress including work organization and job content, and work-life balance could improve the overall mental health and wellbeing of mental health researchers.

Keywords: Mental health, Researchers, Wellbeing, University, Students, Stress

\footnotetext{
*Correspondence: nicole.hill@telethonkids.org.au

${ }^{1}$ Telethon Kids Institute, 15 Hospital Avenue, Nedlands, WA, Australia

Full list of author information is available at the end of the article
} 


\section{Introduction}

The mental health and wellbeing of academic staff and students at higher education institutions (including universities) has become a prominent concern in the research community $[1,2]$. Although there are many benefits and privileges associated with working in academia including knowledge gain, personal fulfilment, flexibility, and comparatively high salaries at senior levels, this career path often involves a range of structural and organisational stressors that may potentially compromise well-being and increase stress. Examples of academicspecific stressors identified in previous studies include being regularly evaluated and 'benchmarked' against output metrics, cyclical competition for funding (including salary support), job insecurity and uncertainty, and balancing multiple roles (teacher, mentor, researcher, writer, reviewer, manager, and administrator) [2-5]. These work commitments regularly interfere with personal life [5, 6] and have been shown in previous meta-analyses to be associated with increased psychological distress, and poor mental health outcomes such as depression and anxiety and suicidal ideation across occupations [7-9].

In response to increased international scrutiny of the academic work culture, several reports have been produced that highlight key work-place stressors experienced by researchers in academic settings $[3,10,11]$. For example, a report published by Wellcome Trust in the United Kingdom [3] highlighted concerns about career uncertainty and longevity, including a culture of long working hours, and continually changing goalposts with overwhelming and unrealistic expectations on productivity. Furthermore, a third of participants (34\%) described accessing support from a mental health professional for depression or anxiety since working in academia [3]. This proportion was higher for females $(38 \%)$ and non-binary respondents (66\%), than males (25\%). However, estimates of symptom prevalence and severity using validated clinical scales (e.g. DASS21) [12] were not collected nor was the association between symptom severity and workrelated psychosocial stressors investigated.

To date, much of what is known about the mental health of academics stems from studies conducted among graduate student populations [13, 14]. However, it is often unclear what proportion of these samples conduct research, with many enrolled in applied study (e.g., medical training) that may not generalise to academics in research roles. Using the Generalized Anxiety Disorder Scale and Patient Health Questionnaire, Evans and colleagues [13] showed graduate research students were more than six times more likely to report experiencing symptoms of mental ill health including anxiety or depression than the general population, with rates of $39 \%$ and $41 \%$ respectively scoring in the 'moderate' or 'severe' range. Furthermore, psychosocial work-related factors such as poor work-life balance and poor mentoring relationships were revealed as being more common in those with a mental health disorder. Another study examined psychological distress [15] using the General Health Questionnaire, as well as job satisfaction, in a large sample of Australian university staff and found that $43 \%$ of academic staff scored above the cut-off [6], indicating increased risk of a possible mental health disorder $[6,16]$. Together these findings suggest that the severity of psychological distress among academics, relative to the general population, may be related to modifiable work-related psychosocial stressors. Given psychological distress is characterised by depressive and anxiety symptoms, and is an indicator of mental ill health conditions such as depression and anxiety disorders [17] research that seeks to identify the association between work-related stresses and psychological distress may have upstream benefits that reduce the progression of a later mental health diagnosis.

Though there is now a growing concern about the mental health of researchers, particularly in early stages of their careers, the majority of work to date has focussed on assessment of stress, environmental factors, or relied on non-clinical instruments to measure researchers' mental health [18]. Additionally, differences in psychological distress and mental health outcomes that are experienced at different career stages (e.g., postgraduate students versus senior researchers) has not been investigated. This gap in evidence is noteworthy since different modifiable work-related stressors may be more or less prevalent at different career stages. Given the link between job stress and the prospective development of psychological distress, mental ill health (e.g., depression and anxiety) and organisational productivity (e.g., sickness, absence rates, and workers compensation claims) [19], understanding the burden of psychological distress and mental ill health, including suicidal ideation, and work-related psychosocial stressors across different career stages has important implications for employees, as well as occupational health, safety regulators, insurers, unions and employers [20].

The current study was undertaken as part of the International Association for Suicide Prevention taskforce on emotional health and wellbeing. It is exploratory in nature, with the aim of investigating the work-related psychosocial stressors experienced by mental health researchers across different career stages, the prevalence of psychological distress and mental ill health, and the association between work-related psychosocial stressors and psychological distress within this population. In doing so, we seek to expand on the existing evidencebase in order to better identify the possible modifiable 
work-related psychosocial stressors that impact mental health researchers and identify possible opportunities for intervention and prevention of psychological distress and mental ill health among mental health researchers.

\section{Material and methods Data and sampling}

This exploratory cross-sectional study used data from an online survey examining the association between workrelated psychosocial stressors and psychological distress in an international sample of specific to mental health researchers. The study was approved by the University of Melbourne Human Research Ethics Committee (ID 1954670). All methods were performed in accordance with the relevant guidelines and regulations and all participants provided informed consent. The survey was available between 28 October 2019 and 1 March 2020 . Participants were recruited via a number of targeted strategies including the email distribution lists of universities and mental health organisations, together with advertisements on the social media platform, Twitter. Recipients were invited to share the link to the study to their potentially eligible colleagues.

Eligible participants included those who were: (1) employed (full time, part time or casually) by a university or research institution (including research assistants, project managers, lecturers, and other academic staff), or; (2) enrolled as a postgraduate student (full-time or part-time Master's or Ph.D. candidate), and; (3) the person's main field of research related to mental health (e.g., psychology, psychiatry, social work). There were no restrictions on geographic location. All participants were screened against the eligibility criteria using an automatic skip-logic algorithm embedded within Qualtrics survey platform [21]. Participants who did not meet the eligibility criteria were not permitted to proceed to the study survey. The survey was formatted so that participants could not complete the survey more than once. Participation was anonymous and participants were not provided any incentive for taking part in the research.

A total of 357 participants provided consent, of whom 207 completed both the Copenhagen Psychosocial Questionnaire III (COPSOQ III) [22] and the Depression Anxiety and Stress Scale (DASS-21), representing 57.2\% of the initial sample. Additional exploratory analyses were conducted on a subsample of 152 participants who answered questions about their history of mental health diagnoses.

\section{Measures}

\section{Sociodemographic and work-related characteristics}

Sociodemographic variables were assessed in a customised survey developed for the purpose of this study and included age, gender (male/female/other), relationship status (entered as a binary variable indicating the presence or absence of a relationship/spouse), dependents (e.g., children including biological and step-children; entered as binary variable representing the presence or absence of dependents), employment status (casual, full-time, part-time), type of work contract (fixed term/ permanent) and clinical (e.g., registered psychologist or doctor) status (yes/no). Participants were classified according to their self-reported career stage. Participants who were employed as a research assistant or project officer were combined into a single category representing support staff. Participants who were enrolled in a Postgraduate degree ( $\mathrm{PhD}$ or Master's degree) were categorised as students. Participants who were employed at postdoctoral level or as a lecturer, were categorised as EMCRs. Lastly, senior researchers were participants who were employed as associate professor or above.

\section{Work-related psychosocial stressors}

Work-related psychosocial exposures were assessed using the COPSOQ III [22]. The COPSOQ III was selected because it has been validated in over 14 countries worldwide [23] and assesses relevant dimensions (e.g., work-life balance) that have been identified as key work-related psychosocial stressors experienced by academics in research settings $[3,5]$. Questionnaire items were obtained from the COPSOQ III middle and core items [24]. The questionnaire used in this study comprised 60 items, encompassing 25 psychosocial dimensions and five domains (Table 1) [22]. Each item is rated on a 5-point Likert scale. All items were transformed to a value ranging between zero (minimum value) to 100 (maximum value) with lower scores representing the lowest probable exposure (never/hardly ever) and 100 representing the highest probable exposure (always or to a very large extent). Higher scores indicated positive outcomes for the work organization and job content, interpersonal relations and leadership, social capital, and general health domains. Whereas higher scores indicated negative outcomes for the demands at work and worklife balance domains. Mean values were summarised according to the five core domains established previously in a previous international validation study that showed acceptable to good reliability with a Cronbach $\alpha>0.7$ [22] and good construct validity [25]. No adaption was made for this study.

\section{General psychological distress}

General psychological distress was assessed using the DASS-21 [12], a self-report measures of depression, anxiety and stress. The DASS- 21 is an internationally validated instrument for measuring psychological distress 
Table 1 COPSOQ III Questionnaire domains and dimensions

\begin{tabular}{|c|c|}
\hline Domains & Dimensions \\
\hline \multirow[t]{4}{*}{ Demands at work } & Emotional demands \\
\hline & Demands for hiding emotions \\
\hline & Quantitative demands \\
\hline & Work pace \\
\hline \multirow[t]{4}{*}{ Work organization and job content } & Influence at work \\
\hline & Possibilities for development \\
\hline & Control over working time \\
\hline & Meaning of work \\
\hline \multirow[t]{9}{*}{ Interpersonal relations and leadership } & Recognition \\
\hline & Predictability \\
\hline & Role conflicts \\
\hline & Role clarity \\
\hline & Illegitimate tasks \\
\hline & Quality of leadership \\
\hline & Social support from supervisor \\
\hline & Social support from colleagues \\
\hline & Sense of community at work \\
\hline \multirow[t]{5}{*}{ Work individual interface (work-life balance) } & Job insecurity \\
\hline & Insecurity over working conditions \\
\hline & Quality of work \\
\hline & Job satisfaction \\
\hline & Work-life conflict \\
\hline \multirow[t]{3}{*}{ Social capital } & Horizontal trust \\
\hline & Vertical trust \\
\hline & Organizational justice \\
\hline General health & Self-rated health \\
\hline
\end{tabular}

[26] and has been shown as a valid and reliable tool for predicting the development of a possible mental health disorder in clinical settings [27]. Participants were asked to score each item on a 4-point Likert scale from 0 (did not apply to me at all) to 3 (applied to me very much). Total scores were computed by adding each item and multiplying the score by a factor 2 [12]. Total scores for the DASS-21 [12] range between zero and 120. Cut-off scores of 60 were labelled high distress [12]. Good interrater reliability, test-retest reliability, and validity of the DASS-21 have been reported previously in both clinical and non-clinical populations [28-30].

\section{Self-reported diagnosed psychological disorder}

Self-reported history of diagnosed mental ill health was assessed using two questions developed specifically for this study: (1) Prior to beginning your research career (including your Ph.D.), have you ever been diagnosed with a psychological disorder? (2) Since beginning your research career (including your PhD), have you ever been diagnosed with a psychological disorder? Participants were provided with the response options 'yes', 'no', and 'I have not been diagnosed, but I probably could have been.'

\section{Suicidal ideation}

Self-reported suicidal ideation was assessed using three questions adapted from item 9 in the Patient Health Questionnaire-9 (PHQ-9; 31). Item 9 in the PHQ-9 [31] evaluates the frequency of suicidal ideation over the preceding two weeks and has been used as a single scale in studies reporting the prevalence of suicidal ideation $[32,33]$ and has shown to be a valid measure of suicidal ideation in studies comparting results with those from detailed clinical interviews [34-36]. In the present study, participants were asked: (1) Over the past two weeks, how often have you been bothered by thoughts that you would be better off dead, or thoughts of hurting yourself in some way? Response options were: Not at all, more than half the days, nearly every day and several days. Items were collapsed into a binary variable representing the presence (consisting of the responses: "more than half the days", "nearly every day" and "several days" or absence (consisting of the response: "not at all") of 
suicidal ideation for each item. Additionally, participants were asked: (2) Over the past year, have you experienced thoughts that you would be better off dead, or thoughts of hurting yourself in some way? and (3) Since beginning your research career (including during your Ph.D.), have you ever experienced thoughts that you would be better off dead, or thoughts of hurting yourself in some way? Participants responded 'yes' or 'no', indicating the presence or absence of suicidal ideation.

\section{Analysis}

Descriptive analysis was conducted to determine the sociodemographic characteristics of the study participants and their history of mental health diagnoses, suicidal ideation, work-related psychosocial exposures and psychological distress. Pearson's chi-square test of independence was used to compare mental health diagnoses and suicidal ideation across career stages (research support staff, postgraduate students, EMCRs, and senior researchers). Group comparisons of work-related psychosocial exposures, DASS-21 [12] psychological distress and related sub-scores were conducted using ANOVA. Multiple pairwise comparisons were performed using the Tukey post hoc test, stratified by career stage.

Multivariate linear regression models [37] were used to estimate the association between the five work-related psychosocial stressor domains and psychological distress, controlling for age, sex, career stage, employment type (fulltime, part-time, casual) and the presence of a mental health policy at work (yes, no, unsure) lifetime mental health-diagnoses (present, absent), suicidal ideation in the past two-weeks (present, absent) for the subsample of 152 participants with complete data. In model 1 the association between work-related psychosocial stressors and psychological distress was adjusted by age and sex (male, female). Model 2 was adjusted for age, sex career stage, hours of employment, employment type, and the presence of a mental health policy at work. Model 3 was adjusted for the covariates included in Model 1 and 2 as well as lifetime mental health diagnosis and suicidal ideation in the past 2 weeks. Coefficients of linear regression $(\beta)$ are calculated and displayed along with their 95\% confidence intervals. To identify differences across models we compared coefficients and confidence intervals to examine whether the models were statistically different. All analyses were conducted in R v 4.1.2.

\section{Results}

Sociodemographic and employment characteristics

Among the 357 participants who provided consent, 207 participants completed the full COPSOQ III [22). survey and DASS-21 [12]; a completion rate of 57.2\%. Participants were from Australia (63.7\%), Europe
(29.9\%), North America (5.3\%), and South East Asia $(<1 \%)$. Most participants were female $(82.1 \%)$ and over half $(56.5 \%)$ were aged $18-34$ years. Table 2 displays the sociodemographic and employment characteristics of participants according to career stage. The largest group of participants were postgraduate students (34.3\%), followed by EMCRs (28.5\%), senior researchers $(20.3 \%)$, with research support staff constituting the smallest group (16.9\%). One third (31\%) of participants reported the presence of a mental health policy at their research institution, however relatively few had read the policy or were aware of its contents (15\%).

\section{Work-related psychosocial exposures}

Table 3 shows the work-related psychosocial exposures according to career stage for the five work-related COPSOQ III [22] domains (see Table 1). Differences between career stages were observed for the domains: 'demands at work', 'work-life balance (termed hereafter as work-life balance) 'social capital' and 'health and wellbeing.' Tukey's post-hoc analysis revealed that the differences in the 'demands at work domain' were driven by lower (i.e., better) scores among research support staff relative to other career stages $(p<0.001$ for postgraduate students, EMCRs and senior researchers, respectively). A similar trend was observed for the 'work-life balance' domain $(p<0.001$ for postgraduate students; $p=0.002$ for EMCRs and $p=0.04$ for senior researchers). Differences in social capital were driven by higher scores among research support staff compared to senior researchers $(p=0.004)$. Lastly, differences in health and wellbeing were driven by higher scores in research support staff compared to postgraduate students $(p=0.003)$ and in senior researchers compared to students $(p=0.03)$.

\section{General psychological distress}

Figures 1, 2 and 3 show sub-scores for depression, anxiety, stress, and total psychological distress measured by the DASS-21 [12], stratified by career stage. Post-hoc comparisons revealed postgraduate students reported experiencing significantly greater anxiety and stress, and total psychological distress compared to research support staff $(p=0.01)$, EMCRs $(p=0.01)$ and senior researchers $(p=0.01$; Table 4$)$. A total of $27(13.4 \%)$ participants reported DASS-21 [12] scores $\geq 60$, indicating severe distress. Severe distress was most frequently reported among postgraduate students $(n=16)$, followed by research support staff $(n=4)$, EMCRs $(n=3)$ and senior researchers $(n=4)$. Fisher's exact test revealed these differences were statistically significant $(p=0.02)$. 
Table 2 Sociodemographic and employment characteristics by career stage

\begin{tabular}{|c|c|c|c|c|c|c|}
\hline & $\begin{array}{l}\text { Total } \\
\mathrm{N}=\mathbf{2 0 7}\end{array}$ & $\begin{array}{l}\text { Support staff } \\
n=35 \\
n(\%)\end{array}$ & $\begin{array}{l}\text { Student } \\
n=71 \\
n(\%)\end{array}$ & $\begin{array}{l}\text { EMCR } \\
n=59 \\
n(\%)\end{array}$ & $\begin{array}{l}\text { Senior researcher } \\
n=42 \\
n(\%)\end{array}$ & $\begin{array}{l}\text { Chi square } \\
p \text { value }\end{array}$ \\
\hline \multicolumn{7}{|l|}{ Gender } \\
\hline Male & 35 & $7(20.0 \%)$ & $8(11.3 \%)$ & $7(11.9 \%)$ & $13(31.0 \%)$ & 0.05 \\
\hline Female & 170 & $28(80.0 \%)$ & 61 (85.9\%) & $52(88.1 \%)$ & $29(69.0 \%)$ & \\
\hline Non-Binary & 2 & - & $2(2.8 \%)$ & - & - & \\
\hline \multicolumn{7}{|l|}{ Age range (years) } \\
\hline $18-24$ & 14 & $8(22.9 \%)$ & $8(11.3 \%)$ & - & - & 0.05 \\
\hline $25-34$ & 103 & $21(60.0 \%)$ & $51(71.8 \%)$ & $31(52.5 \%)$ & - & \\
\hline $35-44$ & 53 & $6(17.1 \%)$ & $7(9.9 \%)$ & $21(35.6 \%)$ & $17(40.5 \%)$ & \\
\hline $45-54$ & 24 & - & $3(4.2 \%)$ & $6(10.2 \%)$ & $15(35.7 \%)$ & \\
\hline $55-64$ & 12 & - & $2(2.8 \%)$ & $1(1.7 \%)$ & $9(21.4 \%)$ & \\
\hline $65+$ & 1 & - & - & - & $1(2.4 \%)$ & \\
\hline \multicolumn{7}{|l|}{ Relationship status } \\
\hline Cohabitating & 52 & $4(11.4 \%)$ & $24(33.8 \%)$ & $19(32.2 \%)$ & $5(11.9 \%)$ & 0.05 \\
\hline Divorced & 2 & - & - & - & $2(4.8 \%)$ & \\
\hline Married & 78 & $6(17.1 \%)$ & $13(18.3 \%)$ & $31(52.5 \%)$ & $28(66.7 \%)$ & \\
\hline Registered partnership & 4 & $2(5.7 \%)$ & $2(2.8 \%)$ & - & - & \\
\hline Separated & 2 & - & $1(1.4 \%)$ & - & $1(2.4 \%)$ & \\
\hline Single & 69 & $23(65.7 \%)$ & $31(43.7 \%)$ & $9(15.3 \%)$ & $6(14.3 \%)$ & \\
\hline \multicolumn{7}{|l|}{ Dependents } \\
\hline Yes & 57 & $4(11.4 \%)$ & $9(12.7 \%)$ & $16(27.1 \%)$ & $28(66.7 \%)$ & 0.00 \\
\hline No & 150 & 31 (88.6\%) & $62(87.3 \%)$ & $43(72.9 \%)$ & $14(33.3 \%)$ & \\
\hline \multicolumn{7}{|l|}{ Clinical degree $^{\mathrm{a}}$} \\
\hline Yes & 45 & $3(8.6 \%)$ & $14(19.7 \%)$ & $15(25.4 \%)$ & $13(31.0 \%)$ & 0.10 \\
\hline No & 157 & $32(91.4 \%)$ & $57(80.3 \%)$ & $45(74.6 \%)$ & 29 (79.0\%) & \\
\hline \multicolumn{7}{|l|}{ Type of work contract } \\
\hline Permanent & 46 & $3(8.6 \%)$ & $6(8.5 \%)$ & $16(27.1 \%)$ & $21(50.0 \%)$ & 0.00 \\
\hline Temporary/Fixed term & 121 & 30 (88.6\%) & $30(42.3 \%)$ & $42(71.2 \%)$ & $19(45.2 \%)$ & \\
\hline Other & 41 & $2(5.7 \%)$ & $36(50.7 \%)$ & $1(1.7 \%)$ & $2(4.8 \%)$ & \\
\hline \multicolumn{7}{|l|}{ Employment status } \\
\hline Full time & 156 & $26(74.3 \%)$ & $47(66.2 \%)$ & $48(81.1$ & $35(83.3 \%)$ & 0.48 \\
\hline Part time & 38 & $7(20.0 \%)$ & 17 (23.9\%) & $9(15.3 \%)$ & $5(11.9 \%)$ & \\
\hline Other & 12 & $2(5.7 \%)$ & $6(8.5 \%)$ & $2(3.4 \%)$ & $2(4.8 \%)$ & \\
\hline \multicolumn{7}{|c|}{ Mental health policy present } \\
\hline Yes & 64 & $16(45.7 \%)$ & $15(30.5 \%)$ & $18(30.5 \%)$ & $15(35.7 \%)$ & 0.06 \\
\hline No & 100 & $11(31.4 \%)$ & $37(52.5 \%)$ & $31(52.5 \%)$ & $21(50.0 \%)$ & \\
\hline Unsure & 43 & $8(22.9 \%)$ & $19(17.0 \%)$ & $10(17.0 \%)$ & $6(14.3 \%)$ & \\
\hline \multicolumn{7}{|c|}{ Participant has read the mental health policy ${ }^{b}$} \\
\hline Yes & 32 & $6(37.5 \%)$ & $7(46.7 \%)$ & $11(61.1 \%)$ & $8(53.3 \%)$ & 0.57 \\
\hline No & 32 & $10(62.5 \%)$ & $8(53.3 \%)$ & $7(38.9 \%)$ & $7(46.7 \%)$ & \\
\hline \multicolumn{7}{|c|}{ Thinks the mental health policy is adequate ${ }^{b}$} \\
\hline Yes & 20 & $3(18.8 \%)$ & $6(40.0 \%)$ & $8(44.4 \%)$ & $3(20.0 \%)$ & 0.48 \\
\hline No & 10 & $2(12.4 \%)$ & $3(20.0 \%)$ & $3(16.7 \%)$ & $2(13.3 \%)$ & \\
\hline Unsure & 34 & $11(68.8 \%)$ & $6(40.0 \%)$ & $7(22.9 \%)$ & $10(66.7 \%)$ & \\
\hline
\end{tabular}

EMCR Early-middle career researcher

${ }^{a}$ Includes participants who are currently completing a clinical degree (e.g., medicine, psychology or similar)

${ }^{b}$ Denominator is based on the number of participants who were aware of their organisation having a mental health policy 
Table 3 COPSOQ III Work-related psychosocial exposures by career stage

\begin{tabular}{|c|c|c|c|c|c|c|c|c|c|}
\hline & \multicolumn{2}{|c|}{ Support staff } & \multicolumn{2}{|c|}{ Student } & \multicolumn{2}{|l|}{ EMCR } & \multicolumn{2}{|c|}{ Senior researcher } & \multirow{2}{*}{$\begin{array}{l}\text { ANOVA } \\
p \text { value }\end{array}$} \\
\hline & Mean & SD & Mean & SD & Mean & SD & Mean & SD & \\
\hline Demands at work & 41.55 & 16.54 & 53.00 & 17.38 & 52.82 & 13.92 & 54.32 & 9.77 & 0.006 \\
\hline Work organization and job content & 71.65 & 12.75 & 73.89 & 16.70 & 73.89 & 11.86 & 75.18 & 9.36 & 0.719 \\
\hline Interpersonal relations and leadership & 68.13 & 11.49 & 64.51 & 16.37 & 62.92 & 13.04 & 62.73 & 12.93 & 0.270 \\
\hline Work-life balance & 40.00 & 11.14 & 49.87 & 11.07 & 48.23 & 10.78 & 48.43 & 10.44 & $<0.001$ \\
\hline Social capital & 66.19 & 16.22 & 57.75 & 23.08 & 56.14 & 16.49 & 51.19 & 17.46 & 0.008 \\
\hline Health and wellbeing & 70.14 & 23.01 & 51.74 & 27.94 & 57.63 & 21.90 & 64.88 & 22.13 & $<0.001$ \\
\hline
\end{tabular}

EMCR Early-middle career researcher

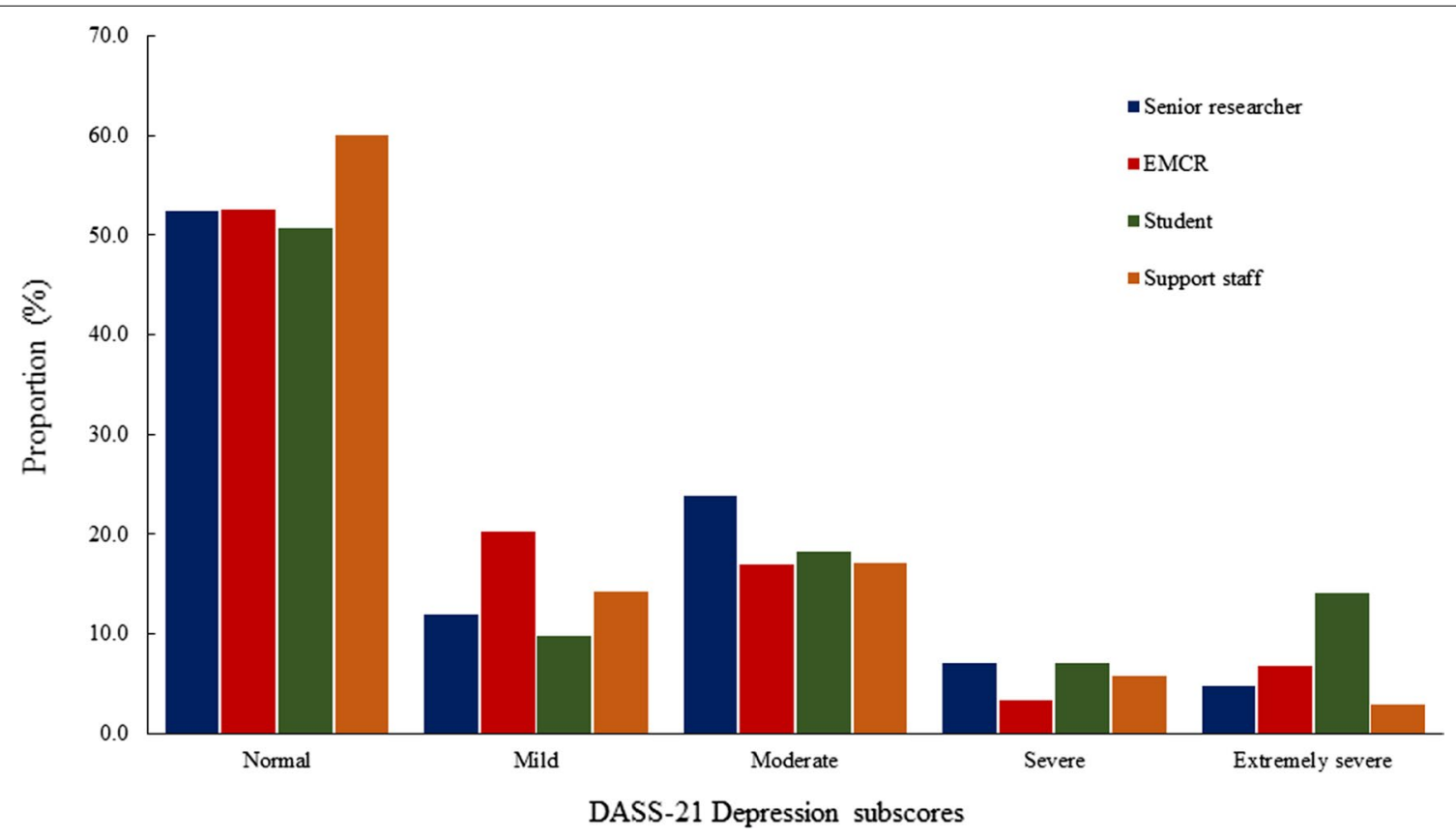

Fig. 1 DASS-21 depression subscores by career stage

\section{Self-reported history of mental health diagnoses and suicidal ideation}

Of the 152 participants who responded to the question on self-reported mental health diagnoses, over half (54.6\%) had received a mental health diagnosis at some point during their lives and a further $46(30.1 \%)$ reported a suspected mental health disorder (i.e., did not receive a diagnosis but thought they should have; Table 5). The proportion of participants who had a diagnosed mental health disorder prior to their academic career was over one-third (37.5\%), while just under one-third (31.6\%) of participants received a psychological diagnosis since commencing their academic career. Senior researchers were significantly less likely to report having received a mental health diagnoses prior to their career in academia, compared to research support staff, postgraduate students, and EMCRs. Of the 80 (52.0\%) participants who reported suicidal ideation since embarking on their academic career, 36 (17.4\%) reported experiencing suicidal ideation in the past fortnight and 69 (33.3\%) reported experiencing suicidal ideation in the past year. All measures of suicidal ideation were comparable across career stages.

\section{Association between work-related psychosocial stressors and psychological distress}

Table 6 shows the results of the regression models examining the relationship between work-related psychosocial exposures and psychological distress. A comparison of the confidence intervals for each of the models included 


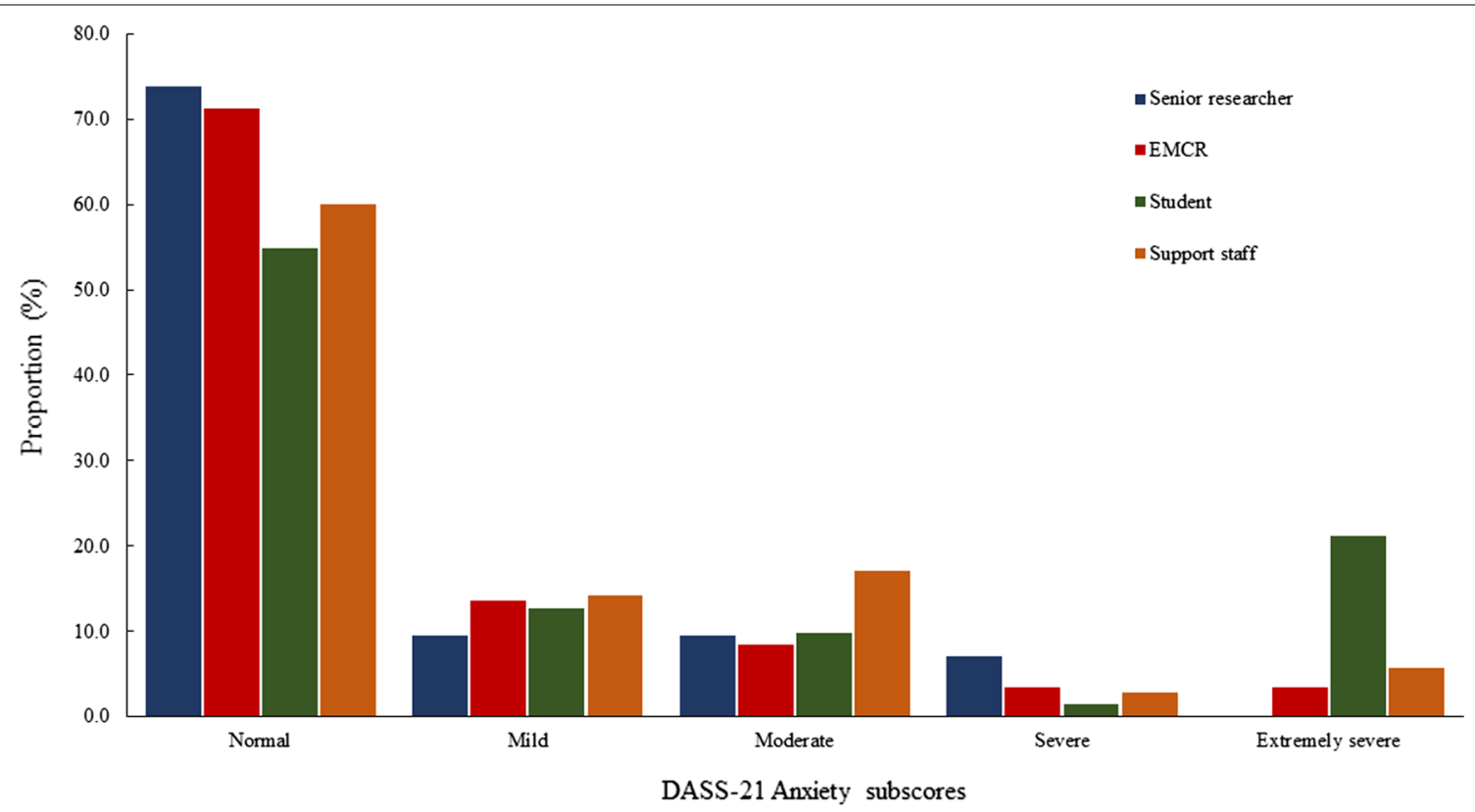

Fig. 2 DASS-21 anxiety subscores by career stage

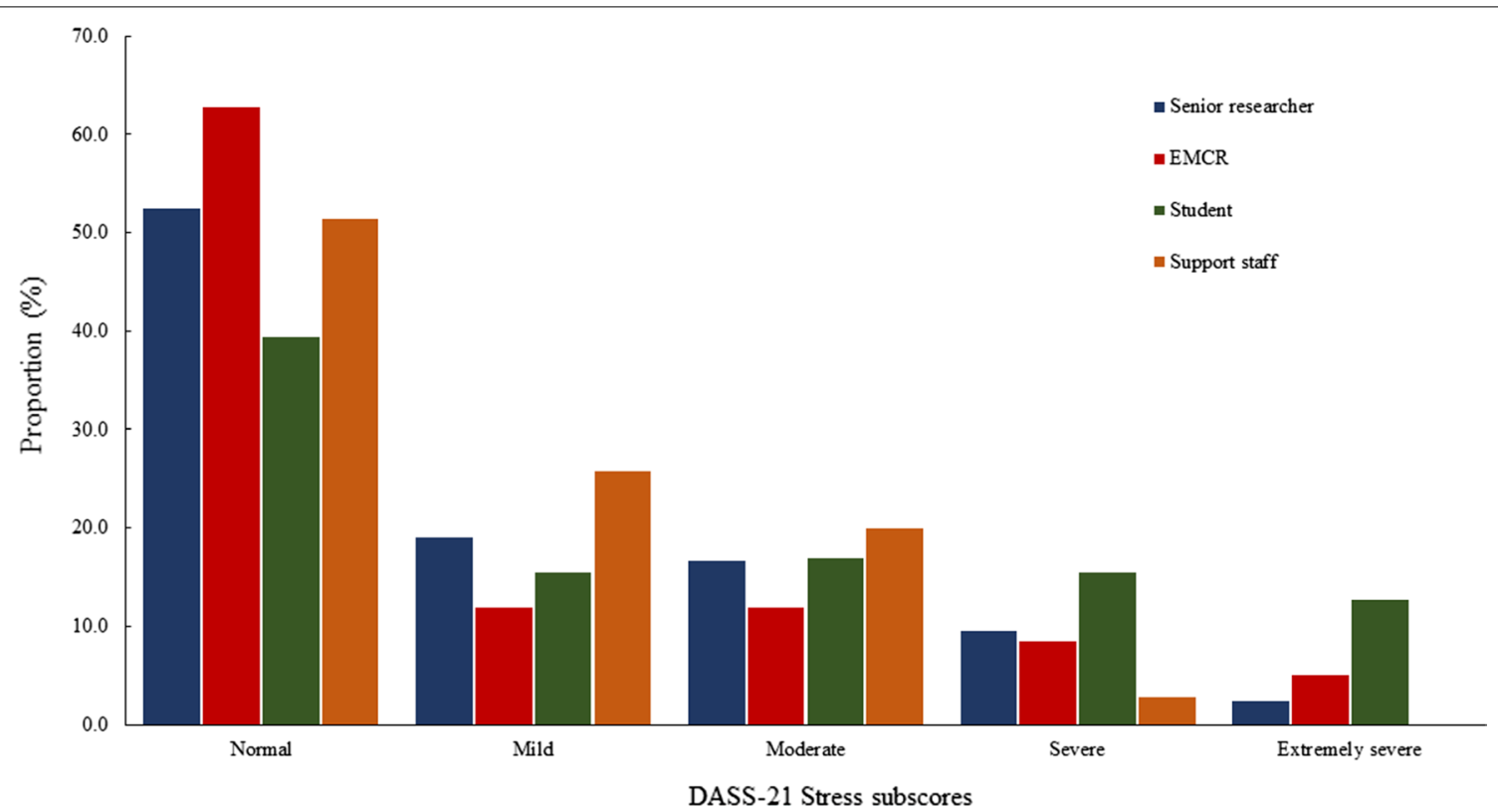

Fig. 3 DASS-21 stress subscores by career stage

in the analysis did not reveal statistically meaningful differences. After adjusting for all covariates, the association between 'interpersonal relations and leadership' and psychological distress was attenuated by the mental health covariates included in model $3(\beta=-0.23, p=0.07)$. The association between the remaining work-related psychosocial domains and psychological distress remained significant. Based on the standardised $\beta$ coefficients from the fully adjusted model (model 3), the strongest associations were observed for 'work organisation and job content' $(\beta=-0.27, p<0.001)$ and 'work-life balance' $(\beta=0.23, p=0.01)$ domains. The weakest association was observed in the social capital dimension $(\beta=-0.10$, $p=0.03)$. 
Table 4 DASS-21 scores stratified by career stage

\begin{tabular}{|c|c|c|c|c|c|c|c|c|c|}
\hline & \multicolumn{2}{|c|}{ Support staff } & \multicolumn{2}{|c|}{ Student } & \multicolumn{2}{|l|}{ EMCR } & \multicolumn{2}{|c|}{ Senior researcher } & \multirow{2}{*}{$\begin{array}{l}\text { ANOVA } \\
p \text { value }\end{array}$} \\
\hline & Mean & SD & Mean & SD & Mean & SD & Mean & SD & \\
\hline Depression & 8.80 & 7.70 & 12.72 & 11.98 & 9.32 & 8.75 & 10.00 & 8.75 & 0.128 \\
\hline Anxiety & 7.03 & 6.35 & 10.37 & 11.15 & 5.49 & 6.34 & 4.62 & 5.35 & $<0.001$ \\
\hline Stress & 12.91 & 7.90 & 19.35 & 10.22 & 13.49 & 9.49 & 14.43 & 8.39 & $<0.001$ \\
\hline Psychological Distress & 28.74 & 18.46 & 42.48 & 29.75 & 28.31 & 20.13 & 29.05 & 18.21 & 0.001 \\
\hline
\end{tabular}

EMCR Early-middle career researcher

Table 5 Mental health history and suicidal ideation by career stage

\begin{tabular}{|c|c|c|c|c|c|c|}
\hline & $\begin{array}{l}\text { Total } \\
\mathrm{N}=152 \\
\mathrm{n}(\%)\end{array}$ & $\begin{array}{l}\text { Support staff } \\
(n=26) \\
n(\%)\end{array}$ & $\begin{array}{l}\text { Student } \\
(\mathrm{n}=57) \\
\mathrm{n}(\%)\end{array}$ & $\begin{array}{l}\text { EMCR } \\
(n=40) \\
n(\%)\end{array}$ & $\begin{array}{l}\text { Senior researcher } \\
(n=29) \\
n(\%)\end{array}$ & $\begin{array}{l}\text { Chi square } \\
p \text { value }\end{array}$ \\
\hline Diagnosed mental health disorder (lifetime) & $83(54.6 \%)$ & $16(61.5 \%)$ & $33(57.9 \%)$ & $23(57.5 \%)$ & $11(37.9 \%)$ & 0.138 \\
\hline Suspected mental health disorder (lifetime) & $46(30.3 \%)$ & $6(23.1 \%)$ & $15(26.3 \%)$ & $15(37.5 \%)$ & $10(34.5 \%)$ & \\
\hline $\begin{array}{l}\text { No diagnosed or suspected mental health disorder (life- } \\
\text { time) }\end{array}$ & $23(15.1 \%)$ & $4(15.4 \%)$ & $9(15.8 \%)$ & $2(5.0 \%)$ & $8(27.6 \%)$ & \\
\hline Diagnosed mental health disorder prior to academic career & $57(37.5 \%)$ & $14(53.8 \%)$ & $26(45.6 \%)$ & $14(35.0 \%)$ & $3(10.3 \%)$ & 0.008 \\
\hline Suspected mental health disorder prior to academic career & $48(31.6 \%)$ & $6(23.1 \%)$ & $13(22.8 \%)$ & $17(42.5 \%)$ & $12(41.4 \%)$ & \\
\hline $\begin{array}{l}\text { No diagnosed or suspected mental health disorder prior to } \\
\text { academic career }\end{array}$ & $47(31.0 \%)$ & $6(23.1 \%)$ & $18(31.6 \%)$ & $9(22.5 \%)$ & $14(48.3 \%)$ & \\
\hline $\begin{array}{l}\text { Diagnosed mental health disorder since beginning aca- } \\
\text { demic career }\end{array}$ & $48(31.6 \%)^{\mathrm{a}}$ & $7(26.9 \%)^{b}$ & $16(28.1 \%)^{c}$ & $16(40.0 \%)^{d}$ & $9(31.0 \%)^{e}$ & 0.478 \\
\hline $\begin{array}{l}\text { Suspected mental health disorder since beginning aca- } \\
\text { demic career }\end{array}$ & $50(32.9 \%)$ & $8(30.8 \%)$ & $18(31.6 \%)$ & $16(40.0 \%)$ & $8(27.6 \%)$ & \\
\hline $\begin{array}{l}\text { No diagnosed or suspected mental health disorder since } \\
\text { beginning academic career }\end{array}$ & $54(35.58 \%)$ & $11(32.3 \%)$ & $23(40.3 \%)$ & $8(20.0 \%)$ & $12(41.4 \%)$ & \\
\hline Suicidal ideation (past 2-weeks) & $36(23.7 \%)$ & $4(15.4 \%)$ & $16(28.1 \%)$ & $8(20 \%)$ & $8(27.6 \%)$ & 0.519 \\
\hline Suicidal ideation (past 12-months) & $69(45.4 \%)$ & $13(50 \%)$ & $26(45.6 \%)$ & $15(37.5 \%)$ & $15(51.7 \%)$ & 0.631 \\
\hline
\end{tabular}

$E M C R$ Early-middle career researcher

${ }^{a} \mathrm{n}=22$ participants who reported mental health diagnoses both prior and since commencing their academic career

${ }^{\mathrm{b}} \mathrm{n}=5$ participants who reported $\mathrm{MH}$ diagnoses both prior and since commencing their academic career

${ }^{c} \mathrm{n}=9$ participants who reported $\mathrm{MH}$ diagnoses both prior and since commencing their academic career

${ }^{d} \mathrm{n}=7$ participants who reported MH diagnoses prior and since commencing their academic career

${ }^{\mathrm{e}} \mathrm{n}=1$ participant who reported MH diagnoses prior and since commencing their academic career

\section{Post-hoc power analysis}

The post-hoc power analysis revealed that with 4 groups, a medium effect size 0.3 , and a power of 0.8 , the recommended sample size for the ANOVA was 44 for each group. The estimated power for the regression analysis was 0.9 , based on 152 participants, 8 covariates, and a medium effect size of 0.3 .

\section{Discussion}

This study sought to describe the psychological distress, mental health and work-related psychosocial stressors experienced by mental health researchers according to their career stage and to identify the association between general psychological distress and work-related psychosocial stressors within the academic settings. Results of the regression analysis provide some insight into the potential modifiable work-related stressors associated with psychological distress among mental health researchers. For example, the strongest associations between psychological distress and work-related psychosocial stressors occurred in the 'work organization and job contents' and 'work-life balance' domains. The 'work organization and job contents' domain include factors such as influence at work, possibilities for development and control over working time, whereas the 'work-life 
Table 6 Multivariate linear regression estimates the COPSOQ III domains and DASS-21 psychological distress outcome

\begin{tabular}{|c|c|c|c|c|c|c|c|c|c|}
\hline & \multicolumn{3}{|l|}{ Model 1} & \multicolumn{3}{|l|}{ Model 2} & \multicolumn{3}{|l|}{ Model 3} \\
\hline & $\beta$ coefficient & $95 \% \mathrm{Cl}$ & $p$ value & $\beta$ coefficient & $95 \% \mathrm{Cl}$ & $p$ value & $\beta$ coefficient & $95 \% \mathrm{Cl}$ & $p$ value \\
\hline Demands at work & 0.192 & & $<0.001$ & 0.155 & & 0.020 & 0.135 & & 0.030 \\
\hline $\begin{array}{l}\text { Work organization } \\
\text { and job content }\end{array}$ & -0.274 & -0.41 to -0.15 & 0.001 & -0.275 & -0.41 to -0.14 & $<0.001$ & 0.275 & -0.36 to -0.11 & $<0.001$ \\
\hline $\begin{array}{l}\text { Interpersonal } \\
\text { relations and } \\
\text { leadership }\end{array}$ & -0.156 & -0.28 to -0.03 & 0.020 & -0.148 & -0.28 to -0.02 & 0.020 & -0.114 & -0.24 to 0.002 & 0.070 \\
\hline Work-life balance & 0.310 & 0.16 to -0.47 & $<0.001$ & 0.272 & 0.11 to 0.44 & 0.001 & -0.229 & 0.07 to 0.39 & 0.010 \\
\hline Social capital & -0.141 & -0.23 to -0.05 & 0.003 & -0.012 & -0.21 to -0.03 & 0.010 & -0.101 & -0.19 to -0.01 & 0.030 \\
\hline $\begin{array}{l}\text { Health and wellbe- } \\
\text { ing }\end{array}$ & -0.204 & -0.27 to -0.13 & $<0.001$ & -0.194 & -0.26 to -0.13 & $<0.001$ & -0.169 & -0.23 to -0.10 & $<0.001$ \\
\hline
\end{tabular}

Model 1 was adjusted for age and sex. Model 2 was adjusted for career stage, hours of employment, employment type, and the presence of a mental health policy at work. Model 3 was adjusted for lifetime mental health diagnosis and suicidal ideation in the past 2 weeks

Cl Confidence interval

balance' domain comprises commitment to the workplace, work engagement, job insecurity, insecurity over working conditions (e.g., office and desk space availability), quality of work, job satisfaction, and work-lifeconflict. The current findings corroborate and extend on those reported in a previous survey involving 4,267 researchers in the UK that showed long-working hours, competing demands which reduce capacity to conduct research, and lack of job security as key concerns faced by academics [3]. Our study extends these findings by showing that after controlling for demographic, employment, and mental health factors, the same work-related psychosocial stressors are associated with increased psychological distress.

Results of the descriptive analysis of mental health and suicidal ideation outcomes revealed that over half of participants had either received a mental health diagnosis in their lifetime or had a suspected mental health diagnosis, compared to approximately $18 \%$ to $36 \%$ reported in previous studies in the general population [38, 39]. Moreover one-third of participants had received a mental health diagnosis since commencing their academic careers. Similarly, rates of suicidal ideation were reported among $52 \%$ of participants, compared to approximately $10 \%$ reported in a previous cross-sectional study of suicidal ideation in the general population [40]. Taken together, these findings suggest that many mental health researchers have lived experience of mental ill health themselves, and that the work-place environment remains an important setting for primary and secondary prevention of mental-ill health.

This study showed that rates of self-reported mental health diagnoses and suicidal ideation were comparable across career stages for those in employment and the post-hoc power analysis demonstrated that these findings are unlikely to be driven by power limitations. However, postgraduate students reported notably higher scores for psychological distress, as well as anxiety, depression, and stress sub-scores, compared to research support staff, EMCRs and senior researchers. Potential explanations include financial strains experienced by many postgraduate students, which may include the need to also engage in paid employment leading to multiple role commitments [41]. Another possibility is that postgraduate students may face greater uncertainty regarding future employment [14, 41]. Indeed, previous studies have shown that although the number of $\mathrm{PhD}$ graduates from science, technology engineering and mathematics has increased substantially over the past 20 -years [42], the number of post-graduate research positions has remained constant, resulting in fewer job prospects among recent graduates [43]. Due to missing data on mental health outcomes it was not feasible to investigate the association between work-related psychosocial stressors and self-reported mental health diagnoses. However previous meta-analytic evidence across occupation groups found factors such as effort-reward imbalance and job insecurity were associated with a 1.81 and 1.91 increased odds of suicidal ideation [8], whereas factors such as long working hours and job insecurity were associated with 1.31-1.77 increased odds of developing an anxiety disorder [8].

It is noteworthy that senior researchers in this study were also significantly less likely to have received a mental health diagnosis prior to their career in academia compared to postgraduate students and EMCRs. On the one hand, it is possible that mental health researchers who stay in academia and transition to senior roles with tenure are those who are less likely to face ongoing work-related stressors that may contribute to their risk of 
psychological distress or mental ill health [44]. It is also possible that students and EMCRs experience significant differences in career pressure and funding success decline that senior researchers did not experience, to the same extent [45].

It has been argued that key structural changes within University institutions such as the marketisation of university education; increased competition between institutions; changes to higher education consumption patterns; the commodification of education; and the growth of managerialism is associated with negative work culture and reduced mental health and wellbeing in recent decades [5]. These structural changes have corresponded with increased student numbers, more demanding students, increased teaching demands, and a shift towards metrics-based performance management [5]. Similar findings were reported in the recent Wellcome trust report into academic work-place culture which identified the tendency for risk aversion and short termism among research institutions, manifested by short term contracts, job insecurity, increased competition to secure limited funding as significant concerns among academic researchers [3]. Moving forward, it is imperative that academic institutions reflect on the impact that structural barriers have on the workplace culture among academics and invest in strategies that have the potential to mitigate the adverse effects associated with psychological distress and wellbeing.

Despite the current recommendations, changes to the institutional structures require time and strategic investment, both of which are unlikely to occur rapidly. Thus it is important that the sector consider interventions that can be implemented in the interim, to bridge the gap between existing work-related psychosocial stressors and wellbeing among academics. Whilst evidence regarding the effectiveness of interventions targeting mental ill health in the workforce is limited, previous studies have shown that screening employees for mental ill health symptoms, proactive outreach, and providing opportunities for therapeutic counselling in the workplace, is both cost effective and associated with improved individual mental health outcomes and workplace productivity [46, 47]. Furthermore, secondary interventions such as stress management, coping, resilience training, mindfulnessbased stress reduction, problem solving, physical activity and cognitive behavioural therapy have been efficacious at increasing productivity and reducing distress in other occupational settings [48-52]. Given less than half of participants in the current study indicated having knowledge of a mental health and wellbeing policy or strategy at their place of employment, an important next-step forward for research institutions is to assess for the presence or absence of mental health and wellbeing policies within the workplace. This includes ensuring that mental health researchers have both access to and knowledge of helpseeking pathways at their institution or place of employment [53] and having policies in place that facilitate employees return to work following an episode of mental ill health [54]. Importantly these policies should include proactive strategies to reduce stigmatizing attitudes and cultures of non-disclosure that have been shown to impact individuals help-seeking behaviours in the workplace [55].

Lastly, data reported in the present study were collected prior to the onset of the COVID-19 pandemic. Factors such as social-distancing restrictions and the transition from office-based to home-based work environments have been linked to disruptions in productivity across disciplines [56]. As such, it is likely that the psychosocial stressors experienced by mental health researchers, such as those involving the work-life balance have increased as a result of COVID-19 restrictions. These effects may be particularly pronounced among specific groups, such as academics with young dependents $[57,58]$ as well as postgraduate students who may have experienced significant disruptions in their social support networks whilst working remotely during their studies. Moving forward, future research that examines the impact of the COVID19 pandemic on the mental health of mental health researchers and academics, more generally, should be prioritised so that decision makers within research institutions can embed timely and appropriate primary and secondary harm minimization strategies, accordingly.

\section{Limitations}

Limitations exist within this study. First, the majority of the sample were from western countries including Australia, UK and USA, with less than 1\% from South East Asia and surrounding geographies. Significant cultural differences may exist in geographic regions not captured by the present survey and remain an important consideration for future studies. Second, the present study was limited to the $57 \%$ of participants who had completed the COPSOQ III [22] questionnaire and selection bias arising from missing data, particularly on suicide ideation outcomes, meant that it was not possible to investigate the association between mental health outcomes and workrelated psychosocial stressors such as job insecurity and suicidal ideation, which have been reported in previous workplace studies [59]. Because attrition was greater than $40 \%$ it was not considered methodologically valid to use statistical adjustments such as multiple imputation on missing data [60]. For this reason the results of the present study should be interpreted in the context of generating hypotheses for future research [60]. 
Third, participants included in this study were selfselected and did not represent a random sample, nor did we sample participants for maximum variation. Furthermore, since participants were recruited via multiple email distribution links and via social media, it was not possible to identify the number of people who were contacted or reached, nor was it possible to calculate rates of refusal. This limitation is means that the study findings may be prone to selection bias and should be interpreted accordingly.

Lastly, previous studies have shown that occupationbased surveys may be susceptible to response biases reflecting higher rates of psychological distress compared to outcomes reported in population-based surveys [61]. This is considered to be a reflection of employees being consciously or unconsciously more inclined to vent their frustrations at their current work [61]. However, Winefield, Gillespie [6] found evidence to suggest that respondents to a university-based survey on stress and psychological distress were neither more nor less likely to display bias in their response based on their current distress. Given the current sample comprises mental health researchers who, by virtue of the academic and mental health training, may be more aware of response biases compared to the general population, we do not expect the results on general psychological distress to be significantly impacted by individual response biases. Nonetheless, as with any self-reported outcomes, results of the present study should be interpreted with caution.

\section{Conclusion}

Over half of mental health researchers have experienced mental ill health during their lives and this figure is greater than those reported in the general population and this warrants concerted efforts to validate these findings against larger, representative samples within academia. Despite working in the same environment, research support staff experience significantly less psychosocial stressors compared to postgraduate students, early-middle career researchers and senior researchers. In contrast, students are significantly more likely to experience mental ill health and suicidal ideation relative to mental health researchers at different career stages. Future research that targets the modifiable stressors at each career stage, including key systemic issues linked to work organization and job content and those that impact work-life balance has the potential to improve the overall mental health and wellbeing of mental health researchers and that these differences ought to be reflected in mental health and wellbeing policy and practice within research institutions.

\section{Acknowledgements}

Not applicable.

\section{Authors' contributions}

NTMH, EB, RB, GC, CW conceived of the research. CG and RB conceived the demographic questionnaire. NTMH, EB and CW were responsible for recruitment. NTMH conducted all data analysis. NTMH, EB, RB, GC, OK, SR, RP, JR, CCW contributed to the manuscript. All authors read and approved the final manuscript.

\section{Funding}

NTMH is funded by a Forrest Research Foundation Prospect Fellowship. GC is funded by the European Council: 101018325, EU 3rd Health Programme and the Health Research Board, Ireland: EIA-2019-005. OJK is supported by a Senior Postdoctoral Fellowship from Research Foundation Flanders (FWO 1257821 N). SR is funded by a Career Development Fellowship from the National Health and Medical Research Council of Australia (GNT1158881), and a Dame Kate Campbell Fellowship from the Faculty of Medicine, Dentistry and Health Sciences at The University of Melbourne. JR is funded by a Career Development Fellowship from the National Health and Medical Research Council of Australia (APP1142348) and the University of Melbourne Dame Kate Campbell Fellowship. CCW is supported by a McKenzie Postdoctoral Research Fellowship at the University of Melbourne (MCK2020292).

\section{Availability of data and materials}

The dataset used and analysed during the current study available from the corresponding author on reasonable request.

\section{Declarations}

\section{Ethics approval and consent to participate}

Human subjects' approval and all information was received by the University of Melbourne Human Research Ethics Committee for the key informant interviews (ID: 1954670). Written informed consent was collected from all participants. It was emphasized that participation was voluntary. All methods were performed in accordance with the relevant guidelines.

\section{Consent for publication}

Participants were informed that the information they gave in the survey would be published in anonymised form.

\section{Competing interests}

The authors declare no competing interests.

\section{Author details}

${ }^{1}$ Telethon Kids Institute, 15 Hospital Avenue, Nedlands, WA, Australia. ${ }^{2}$ Centre for Child Health Research, The University of Western Australia, Perth, Australia. ${ }^{3}$ School of Population and Global Health, University of Western Australia, Perth, Australia. ${ }^{4}$ Orygen, Parkville, Melbourne, Australia. ${ }^{5}$ Centre for Youth Mental Health, The University of Melbourne, Parkville, Australia. ${ }^{6}$ School of Public Health, University College Cork, Cork, Ireland. ${ }^{7}$ National Suicide Research Foundation, Cork, Ireland. ${ }^{8}$ Department of Neuroscience, Center for Contextual Psychiatry, KU Leuven, Leuven, Belgium.

Received: 4 August 2021 Accepted: 25 January 2022

Published online: 01 February 2022

\section{References}

1. Woolston C. Feeling overwhelmed by academia? You are not alone. Nature. 2018;557(7703):129.

2. Chhabra A. Mental health in academia is too often a forgotten footnote. That needs to change USA: Science; 2018. Available from: https:// www.science.org/content/article/mental-health-academia-too-oftenforgotten-footnote-needs-change. Date Accessed April 2021.

3. Wellcome Trust. What researchers think about the culture they work in. UK: Wellcome Trust; 2020.

4. Roach $\mathrm{M}$, Sauermann $\mathrm{H}$. The declining interest in an academic career. PLOS ONE. 2017;12(9):e0184130.

5. Sang K, Powell A, Finkel R, Richards J. Being an academic is not a 9-5 job': long working hours and the 'ideal worker UK academia. Labour Ind. 2015;25(3):235-49. 
6. Winefield AH, Gillespie N, Stough C, Dua J, Hapuarachchi J, Boyd C. Occupational stress in Australian university staff: results from a national survey. Int J Stress Manag. 2003;10(1):51-63.

7. Duchaine CS, Aubé K, Gilbert-Ouimet M, Vézina M, Ndjaboué R, Massamba V, et al. Psychosocial stressors at work and the risk of sickness absence due to a diagnosed mental disorder: a systematic review and meta-analysis. JAMA Psychiat. 2020;77(8):842-51.

8. Niedhammer I, Bertrais S, Witt K. Psychosocial work exposures and health outcomes: a meta-review of 72 literature reviews with meta-analysis. Scand J Work Environ Health. 2021;7:489-508.

9. van der Molen HF, Nieuwenhuijsen K, Frings-Dresen MHW, de Groene G. Work-related psychosocial risk factors for stress-related mental disorders: an updated systematic review and meta-analysis. BMJ Open. 2020;10(7):e034849

10. Acton SE, Bell AJD, Toseland CP, Twelvetrees A. A survey of new PIs in the UK. eLife. 2019;8:e46827.

11. McConnell SC, Westerman EL, Pierre JF, Heckler EJ, Schwartz NB. United States National Postdoc Survey results and the interaction of gender, career choice and mentor impact. eLife. 2018;7:189.

12. Lovibond S, Lovibond PF. Manual for the depression anxiety stress scales. 2nd ed. Sydney: Psychology Foundation; 1995.

13. Evans TM, Bira L, Gastelum JB, Weiss LT, Vanderford NL. Evidence for a mental health crisis in graduate education. Nat Biotechnol. 2018;36:282.

14. Woolston C. Graduate survey: a love-hurt relationship. Nature. 2017:550(7677):549-52.

15. Huppert FA. Psychological well-being: evidence regarding its causes and consequences. App Psychol Health Wellbeing. 2009:1(2):137-64.

16. Anjara SG, Bonetto C, Van Bortel T, Brayne C. Using the GHQ-12 to screen for mental health problems among primary care patients: psychometrics and practical considerations. IJMHS. 2020;14(1):62.

17. Mirowsky J, Ross CE. Measurement for a Human Science. J Health Soc Behav. 2002;43(2):152-70.

18. Guthrie S, Lichten CA, Van Belle J, Ball S, Knack A, Hofman J. Understanding mental health in the research environment. Rand Health Q. 2018;7(2).

19. Stephen S, Bridget C. Psychosocial work environment and mental healtha meta-analytic review. Scand J Work Environ Health. 2006;32(6):443-62.

20. LaMontagne AD, Keegel T, Vallance D. Protecting and promoting mental health in the workplace: developing a systems approach to job stress. Health Promot J Austr. 2007;18(3):221-8.

21. Qualtrics. 2005 ed. Provo, Utah, USAFirst release: 2005, copyright year 2021.

22. Burr H, Berthelsen $H$, Moncada S, Nübling M, Dupret E, Demiral Y, et al. The third version of the copenhagen psychosocial questionnaire. Saf Health Work. 2019;10(4):482-503.

23. COPSOQ International Network. Validation studies Breisgau, Germany 2020. Available from: https://www.copsoq-network.org/validation-studi es/. Date Accessed December 2020

24. Llorens C, Pérez-Franco J, Oudyk H, Berthelsen E, Dupret M, Nübling H, et al. COPSOQ III. Guidelines and questionnaire. Denmark: COPSOQ III International Network; 2019

25. Bjorner JB, Pejtersen JH. Evaluating construct validity of the second version of the Copenhagen Psychosocial Questionnaire through analysis of differential item functioning and differential item effect. Scand J Public Health. 2010;38(3 Suppl):90-105.

26. Zanon C, Brenner RE, Baptista MN, Vogel DL, Rubin M, Al-Darmaki FR, et al. Examining the dimensionality, reliability, and invariance of the Depression, Anxiety, and Stress Scale-21 (DASS-21) across eight countries. Assessment. 2021:28(6):1531-44.

27. Beaufort IN, De Weert-Van Oene GH, Buwalda VA, de Leeuw JRJ, Goudriaan AE. The depression, anxiety and stress scale (DASS-21) as a screener for depression in substance use disorder inpatients: a pilot study. Eur Addict Res. 2017:23(5):260-8.

28. Gloster AT, Rhoades HM, Novy D, Klotsche J, Senior A, Kunik M, et al. Psychometric properties of the Depression Anxiety and Stress Scale-21 in older primary care patients. J Affect Disord. 2008; 1 10(3):248-59.

29. Osman A, Wong JL, Bagge CL, Freedenthal S, Gutierrez PM, Lozano G. The Depression Anxiety Stress Scales-21 (DASS-21): further examination of dimensions, scale reliability, and correlates. J Clin Psychol. 2012;68(12):1322-38
30. Henry JD, Crawford JR. The short-form version of the Depression Anxiety Stress Scales (DASS-21): construct validity and normative data in a large non-clinical sample. Br J Clin Psychol. 2005;44(2):227-39.

31. Kroenke K, Spitzer RL, Williams JB. The PHQ-9: validity of a brief depression severity measure. J Gen Intern Med. 2001;16(9):606-13.

32. Walker J, Hansen CH, Butcher I, Sharma N, Wall L, Murray G, et al. Thoughts of death and suicide reported by cancer patients who endorsed the "Suicidal Thoughts" Item of the PHQ-9 during routine screening for depression. Psychosomatics. 2011;52(5):424-7.

33. Simon GE, Rutter CM, Peterson D, Oliver M, Whiteside U, Operskalski $\mathrm{B}$, et al. Does response on the PHQ-9 Depression Questionnaire predict subsequent suicide attempt or suicide death? Psychiatr Serv. 2013;64(12):1195-202.

34. Walker J, Hansen CH, Hodges L, Thekkumpurath P, O'Connor M, Sharma $\mathrm{N}$, et al. Screening for suicidality in cancer patients using Item 9 of the nine-item patient health questionnaire; does the item score predict who requires further assessment? Gen Hosp Psychiatry. 2010;32(2):218-20.

35. Uebelacker LA, German NM, Gaudiano BA, Miller IW. Patient health questionnaire depression scale as a suicide screening instrument in depressed primary care patients: a cross-sectional study. Prim Care Companion CNS Disord. 2011;13(1):PCC.10m01027.

36. Razykov I, Hudson M, Baron M, Thombs BD, Group CSR. Utility of the Patient Health Questionnaire-9 to assess suicide risk in patients with systemic sclerosis. Arthritis Care Res. 2013;65(5):753-8.

37. Schneider A, Hommel G, Blettner M. Linear regression analysis: part 14 of a series on evaluation of scientific publications. Dtsch Arztebl Int. 2010;107(44):776-82.

38. Kessler RC, Aguilar-Gaxiola S, Alonso J, Chatterii S, Lee S, Ormel J, et al. The global burden of mental disorders: an update from the WHO World Mental Health (WMH) surveys. Epidemiol Psichiatr Soc. 2009;18(1):23-33.

39. Steel Z, Marnane C, Iranpour C, Chey T, Jackson JW, Patel V, et al. The global prevalence of common mental disorders: a systematic review and meta-analysis 1980-2013. Int J Epidemiol. 2014;43(2):476-93.

40. Nock MK, Borges G, Bromet EJ, Alonso J, Angermeyer M, Beautrais A, et al. Cross-national prevalence and risk factors for suicidal ideation, plans and attempts. Br J Psychiatry. 2008;192(2):98-105.

41. Watson SJ, Barber BL, Dziurawiec S. The role of economizing and financial strain in Australian University Students' psychological well-being. J Fam Econ Issues. 2015;36(3):421-33.

42. Cyranoski D, Gilbert N, Ledford H, Nayar A, Yahia M. Education: the PhD factory. Nature. 2011;472(7343):276-9.

43. Schillebeeckx M, Maricque $B$, Lewis $C$. The missing piece to changing the university culture. Nat Biotechnol. 2013;31(10):938-41.

44. Crick KA, Larson LM, Seipel MT. Non-tenure track faculty satisfaction: a self-determination model. J Career Assess. 2019;28(3):425-45.

45. Johnson AP, Lester RJ. Mental health in academia: hacks for cultivating and sustaining wellbeing. Am J Hum Biol. 2021:e23664.

46. Wang PS, Simon GE, Avorn J, Azocar F, Ludman EJ, McCulloch J, et al. Telephone screening, outreach, and care management for depressed workers and impact on clinical and work productivity outcomes: a randomized controlled trial. JAMA. 2007;298(12):1401-11.

47. Schene AH, Koeter MW, Kikkert MJ, Swinkels JA, McCrone P. Adjuvant occupational therapy for work-related major depression works: randomized trial including economic evaluation. Psychol Med. 2007:37(3):351-62.

48. Ornek OK, Esin MN. Effects of a work-related stress model based mental health promotion program on job stress, stress reactions and coping profiles of women workers: a control groups study. BMC Public Health. 2020;20(1):1658.

49. Arapovic-Johansson B, Jensen I, Wåhlin C, Björklund C, Kwak L. Process evaluation of a participative organizational intervention as a stress preventive intervention for employees in Swedish Primary Health Care. Int J Environ Res Public Health. 2020;17(19):7285.

50. Janssen M, Heerkens Y, Kuijer W, van der Heijden B, Engels J. Effects of mindfulness-based stress reduction on employees' mental health: a systematic review. PLOS ONE. 2018;13(1):e0191332.

51. de Bruin El, Formsma AR, Frijstein G, Bögels SM. Mindful2Work: effects of combined physical exercise, yoga, and mindfulness meditations for stress relieve in employees A Proof of Concept Study. Mindfulness (N Y). 2017:8(1):204-17. 
52. Sun Y, Wang A, Yu S, Hagger MS, Chen X, Fong SSM, et al. A blended intervention to promote physical activity, health and work productivity among office employees using intervention mapping: a study protocol for a cluster-randomized controlled trial. BMC Public Health. 2020;20(1):994.

53. World Health Organisation. Mental health and work: Impact issues and good practices. Geneva; 2000.

54. Reavley NJ, Ross A, Killackey EJ, Jorm AF. Development of guidelines to assist organisations to support employees returning to work after an episode of anxiety, depression or a related disorder: a Delphi consensus study with Australian professionals and consumers. BMC Psychiatry. 2012;12(1):135

55. King AJ, Brophy LM, Fortune TL, Byrne L. Factors affecting mental health professionals'sharing of their lived experience in the workplace: a scoping review. Psychiatr Serv. 2020;71(10):1047-64.

56. Arora VM, Wray CM, O'Glasser AY, Shapiro M, Jain S. Leveling the playing field: accounting for academic productivity during the COVID-19 pandemic. J Hosp Med. 2021;16(2):120-3.

57. Delaney RK, Locke A, Pershing ML, Geist C, Clouse E, Precourt Debbink M, et al. Experiences of a health System's Faculty, Staff, and Trainees' career development, work culture, and childcare needs during the COVID-19 pandemic. JAMA Netw Open. 2021;4(4):e213997.

58. Cardel MI, Dean N, Montoya-Williams D. Preventing a secondary epidemic of lost early career scientists. Effects of COVID-19 pandemic on women with children. Ann Am Thorac Soc. 2020;17(11):1366-70.

59. Kim W, Ki M, Choi M, Song A. Comparable risk of suicidal ideation between workers at precarious employment and unemployment: data from the Korean Welfare Panel Study, 2012-2017. Int J Environ Res Public Health. 2019;16(16):2811.

60. Jakobsen JC, Gluud C, Wetterslev J, Winkel P. When and how should multiple imputation be used for handling missing data in randomised clinical trials - a practical guide with flowcharts. BMC Med Res Methodol. 2017;17(1):162.

61. Goodwin L, Ben-Zion I, Fear NT, Hotopf M, Stansfeld SA, Wessely S. Are reports of psychological stress higher in occupational studies? A systematic review across occupational and population based studies. PLOS ONE. 2013;8(11):e78693.

\section{Publisher's Note}

Springer Nature remains neutral with regard to jurisdictional claims in published maps and institutional affiliations.

Ready to submit your research? Choose BMC and benefit from:

- fast, convenient online submission

- thorough peer review by experienced researchers in your field

- rapid publication on acceptance

- support for research data, including large and complex data types

- gold Open Access which fosters wider collaboration and increased citations

- maximum visibility for your research: over 100M website views per year

At BMC, research is always in progress.

Learn more biomedcentral.com/submissions 